\title{
One stage transanal full thickness pull-through operation for rectosigmoid Hirschsprung's disease
}

\author{
A. K. M. Zahid Hossain, Gazi Zahirul Hasan, Susankar Kumar Mandal, Md. Nooruzzaman, \\ A. Shahinoor and Dinesh Prasad Koirala
}

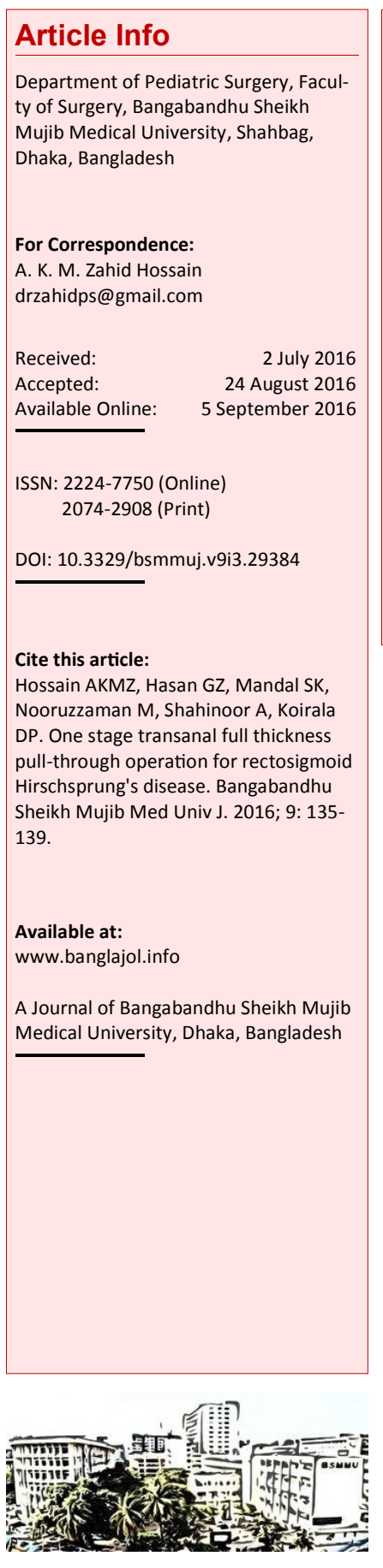

\begin{abstract}
The surgical management of Hirschsprung's disease (HD) has changed from the original staged operations to the latest introduced minimally invasive one stage techniques. One stage transanal full thickness Swenson-like procedure is a new concept of single stage procedure for HD. We reviewed the early outcome of one stage transanal Swenson-like pull-through operation for rectosigmoid HD. By using the transanal concept we choose to apply Swenson's principle in transanal dissection for the primary treatment of HD and describe technical aspects and impact on fecal and urinary function. We reviewed our series of HD patient who underwent one-stage transanal full thickness, Swenson-like rectosigmoid dissection, assessing for postoperative stricture or stenosis, anastomotic leak, enterocolitis, obstruction and long-term results for bowel and urinary function. Of 15 patients all had the transanal resection, the age of the patients ranged from 6 months to 5 years. The average length of resection was $20 \pm 5 \mathrm{~cm}$. Mean follow-up was 24 months. Ten patients were at least three years old at follow-up and were assessed for urinary and fecal continence. All had the voluntary bowel movement and urinary continence. Three patients had episodes of postoperative enterocolitis and two patients developed stenosis at the anastomotic site. Postoperative frequent bowel movement was present in all patients and continued for 2-8 weeks. In conclusion, our data support the fact that a modification of Swenson's original transabdominal dissection concept using the recently describe transanal approach is an excellent technique for HD and produces excellent long-term outcome for fecal and urinary function.
\end{abstract}

\section{Introduction}

It has been more than 70 years since the original description of the etiology of Hirschsprung's disease (HD) elucidated by Dr. Ovar Swenson.1 Children who suffered from HD have chronic constipation, enterocolitis, failure to thrive, abdominal distension and dependent on assisted defecation, an initial colostomy was usually done. It was followed by pull-through operation and subsequent closure of colostomy done. The surgical management of rectosigmoid HD recently changed from staged procedure to a one stage transanal pull-through operation. The pull-through operation originated from Swenson full thickness dissection,? Soave's endorectal dissection, $\underline{\underline{3}}$ Duhamel's retrorectal dissection and pouch procedure, $\underline{4}$ and Rehbein's low anterior resection, $\underline{\mathbf{5}}$ to more recently introduced primary single stage transanal procedure, $6-8$ Georgeson's minimal access technique. 9 The goal of surgical management for HD are to remove the aganglionic bowel and reconstruct the intestinal tract by bringing the normally innervated bowel down to the anus while preserving normal sphincter function and the anal canal. The surgical management of the rectosigmoid HD is rapidly changing from the three stage procedure to a single stage, transanal, pull-through procedure. Since De La Torre and Ortega-Salgado 7 and Langer et als published their reports in the late 1990s, one stage transanal pull-through procedure for HD become popular. The single stage transanal approach has the principal advantage of avoiding the transabdominal mobilization of the colon and the rectum. $\underline{10,11}$ Transanal procedure leave no scars, has less postoperative pain, shorter hospital stay, reducing the cost and the morbidity associated with the staged procedure.

Most commonly used transanal approach is endorectal pull-through, which leaves a long muscular cuff and usually split longitudinally either posteriorly or anteriorly.., 12 It is wellknown that the long muscular cuff that is left behind causes obstructive symptoms and enterocolitis. A few studies in the literature have shown that the problem of the remnant rectal cuff can be avoided if the transanal resection of the aganglionic segment is perfor- 
med in the manner described by Swenson, by dissecting the full thickness of the rectum.13,14

In this article, we will demonstrate our experience with transanal, full thickness Swenson-like dissection for rectosigmoid HD, which uses the experience with the full thickness rectal wall dissection of anorectal malformation and using the idea of the transanal approach.15-17 The Swenson transabdominal approach became less popular due to wide dissection made by some of the surgeons which cause many complications. With an elegant technique in the proper plane, we feel that this approach is safe, reproducible and preserves urinary, fecal and sexual functions. We also like this procedure because it allows complete removal of the entire aganglionic gut without leaving a muscular cuff or a pouch.

\section{Materials and Methods}

From January 2012 to December 2015 about 15 children with rectosigmoid HD have done the transanal single stage full thickness pull-through operation (transanal Swenson-like approach). All the patients presented with a history of delayed passage of meconium and constipation and the rectosigmoid $\mathrm{HD}$ was diagnosed by a contrast enema (Figure 1) and rectal biopsy. Patients characteristics were collected and included age, gender, associated anomalies and other medical problems. We excluded patients with significantly associated anomalies, also excluded the patient with ostoma and total colonic HD. Information on early and late postoperative complications was evaluated as well as long-term outcomes regarding fecal and urinary continence. Rates of stricture formation, pelvic abscess, constipation, and enterocolitis were noted as well.

The technique for rectal dissection was a transanal, full thickness, Swenson-like approach with careful preservation of the dentate line. Intraoperative frozen section biopsy was used to assess that the pull-through segment has ganglionic cells and no thickened nerve fibers. We also collected data regarding the length of the each resected specimen.

Patient's hospital stay and follow-up at outpatient department at 15 days, 1 month, 3 months and 6 months and yearly were evaluated. Data was expressed as mean and range $\pm \mathrm{SD}$.

\section{Surgical technique}

The pre-operative preparation was done with rectal irrigations and antibiotics. Under general anesthesia, all patients underwent total body preparation, a per-urethral catheterization was placed and the rectum was irrigated with dilute povidone iodine solution. In prone, buttock elevated position, the perianal retraction was done with 6 to 8 circum- ferential silk sutures (Figure 2). Also, some silk suture was given just proximal to the dentate line so that was no longer visible; assuring that the dentate line would be protected during dissection. In this was the distal $1.5 \mathrm{~cm}$ of the anal canal is preserved. Interrupted fine silk suture was placed 1 to $1.5 \mathrm{~cm}$. proximal to the dentate line in a circumferential fashion to provide uniform traction. At the beginning, a full thickness incision was made on the rectal wall posteriorly along traction line of silk suture. The incision was extended all around the circumference, incising the full thickness of the rectal wall. Very precise coagulation of vessels right along the rectal wall was performed. Rectal mobilization was done by working on the surface of the rectal wall similar to the mobilization performed for the anorectal malformation using electrocautery. The dissection was carried into peritoneal cavity and dissection could be done easily once peritoneal reflection was reached allowing mobilization of the rectum and sigmoid colon out of the anus without leaving behind any muscular cuff. The proximal dissection was continued till the transition zone was visible and the colon was pulled through to the appropriate level, confirmed by intra-operative frozen section biopsy. Full thickness biopsy was sent to pathology assuring analysis of the muscularis and submucosa. It is important to note that the vessels should be coagulated or divided after suture ligation just before they enter the intestinal wall to avoid injury to pelvic vessels and nerves including the prostate or vagina. As the dissection progress proximally to maintain the orientation of the gut marking silk suture given at 6 'o clock position which prevents twisting of the gut. The aganglionic colonic segment was resected and a full thickness colo-anal anastomosis was completed in two layers $1.0 \mathrm{~cm}$ proximal to the dentate line.

It is very important to careful not to include the dentate line during colo-anal anastomosis as they may produce pain and may lead to fecal incontinence.

\section{Results}

A total of 15 children, 10 male and 5 female with age ranging from 6 months to 5 years (median 18 months) underwent one stage transanal full thickness pull-through (transanal Swenson procedure). We performed a purely transanal resection for the patients without preoperative diversion who had a well-defined sigmoid (or lower) transition zone on contrast enema. The operating time, including the time taken for frozen sections, ranged from 120 to $180 \mathrm{~min}$ (mean $140 \mathrm{~min}$ ). The length of the bowel resection ranged from 15 to $25 \mathrm{~cm}$ (mean $20 \mathrm{~cm}$ ).

Operative complications were evaluated. There were 3 patients who required a blood transfusion. 


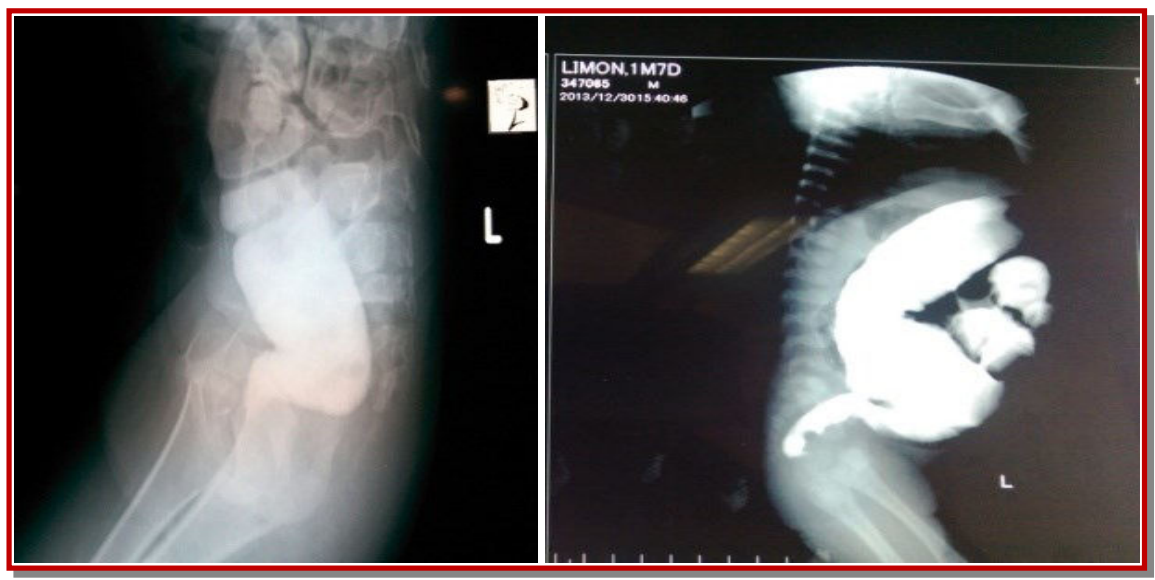

Figure 1: Barium enema examination in different children demonstrate Hirschsprung disease. The aganlonic rectum was small and contracted. The proximal colon was dilated. A transitional zone between aganlonic and ganglionic colon was nicely seen
Postoperatively 5 patients passed stool within 24 hours and other 10 had bowel movements within two to three days of the operation. All the 15 patients tolerated full oral food by the 4th postoperative day. The hospital stay ranged from 6-8 days with median 7 days. There were no intra-operative or post operated complication related to the pull-through procedure. None of the children had an anastomotic leak, anastomotic dehiscence, wound infections or bowel obstruction. Three patients had postoperative enterocolitis, which resolved with antibiotics and rectal irrigation. Two children developed anastomotic stenosis. The stenosis was evident by the sixth to eighth postoperative weeks and required anal dilatation. The follow-up period ranges from 6 to 36 months (mean 24 months).

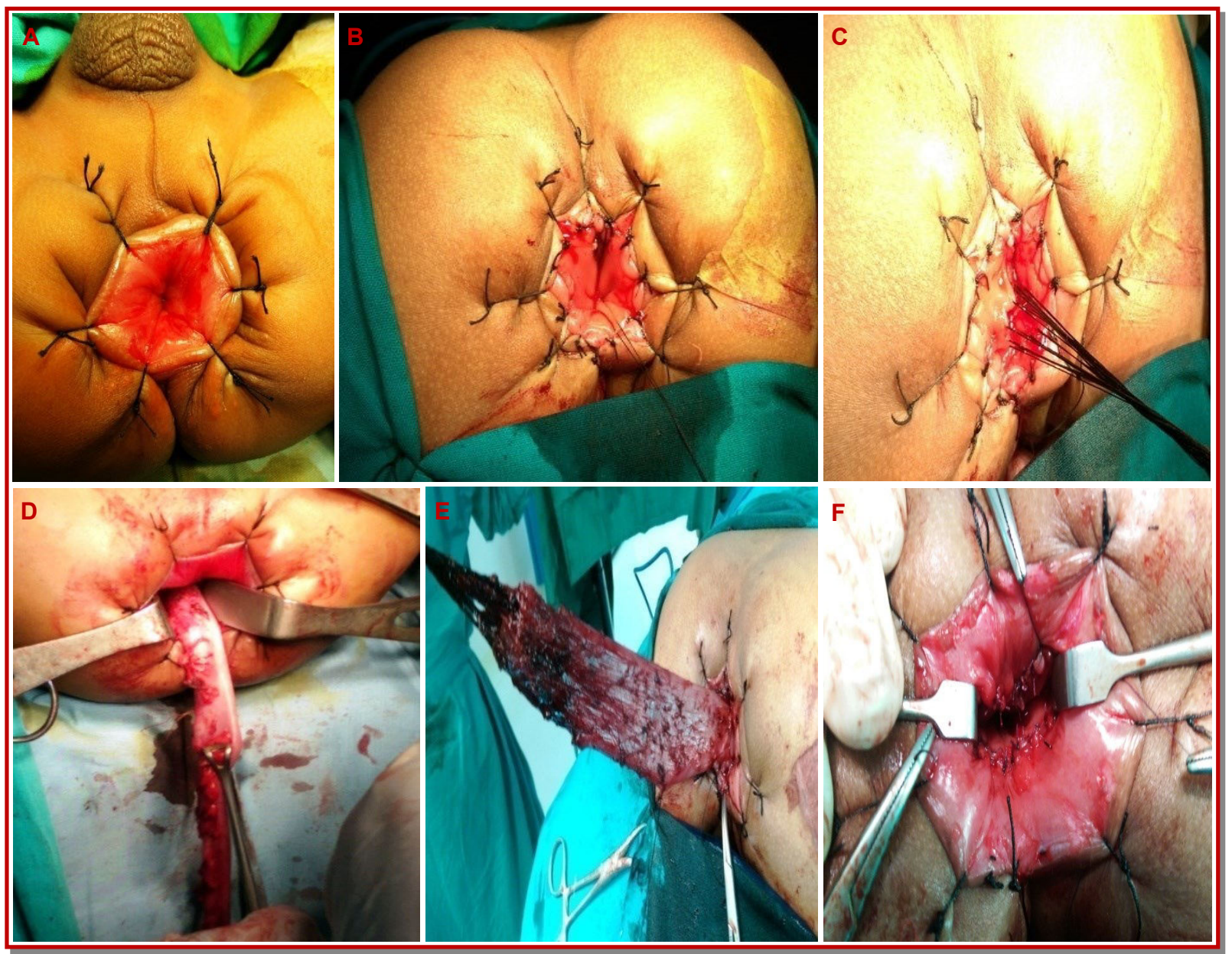

Figure 2: Perianal retraction was done with 6 to 8 circumferential silk sutures (A); Silk suture was given just proximal to the dentate line so that was no longer visible. Assuring that the dentate line would be protected during dissection (B); Interrupted fine silk sutures were placed 1 to $1.5 \mathrm{~cm}$ proximal to the dentate line in a circumferential fashion to provide uniform traction (C); Dissection was carried in to peritoneal cavity and dissection could be done easily once peritoneal reflection was reached allowing mobilization of the rectum and sigmoid colon out of the anus without leaving behind any muscular cuff (D); Incision was extended all around the circumference, incising the full thickness of the rectal wall (E); Full thickness colo-anal anastomosis was completed in two layers (F) 
Postoperative frequent bowel movement and soiling were seen in most of the patients which usually disappear after two to eight weeks (mean 4 weeks). Most of the patients $(12 / 15,80 \%)$ had achieved normal bowel movements within four weeks. No voiding disturbance in the form of incontinence, poor stream or retention was encountered during the follow-up period and the ultrasound examination of the urinary bladder did not reveal any post-void residual urine. The initial postoperative frequent bowel movements reduced to daily two to three bowel movements by the 4th week and no patient complained of recurrent constipation. The postoperative pain was minimum and all the patients could be started on oral feeding after 48 hours.

\section{Discussion}

The first report on the transanal procedure for the classic recto-sigmoid HD was published in 1998. The primary transanal pull-through techniques with various modification have become the standard of care around the world, each having their own proponents. 18 The advantages of one stage transanal procedures are avoidance of multiple laparotomies and preliminary colostomy with their associated complications, reduced operating time, less blood loss, no pelvic structure damage, short hospital stay, decreased need for analgesics, significantly lower hospital cost, no external scars and with improved cosmetic appearance, $, 9,12$ without compromising on the functional outcomes. 19 Above all, there is a high degree of parental acceptance of the procedure, as it avoids the need to care for a baby with a colostomy. However, the aganglionic rectal muscular cuff in the transanal Soave's procedure limits the volume of the replaced sigmoid and rectum. $\underline{20}$ This remnant rectal cuff also causes cuff strictures, obstruction, enterocolitis and incontinence. To prevent these complications many authors have recommended splitting the muscular cuff posteriorly up to the sphincter to prevent obstruction of the pulled through the gut. The cuff can be rolled down during pull-through of the gut and may form a ring-like constriction just above the colo-anal anastomosis.21 To prevent this problem many authors did shorter mucosectomy with a shorter muscle cuff about 1-2 $\mathrm{cm}$ above dentate line.22 Some authors have completely avoided the submucosal dissection and describe a transanal Swenson-like procedure without keeping any residual muscular cuff. $\underline{\underline{13}}$

We have performed one stage transanal full thickness pull-through (transanal Swenson procedure) in selected cases after confirmation of diagnosis by contrast enema and full thickness rectal biopsy. Intra-operatively, we started our full thickness rectal dissection $1 \mathrm{~cm}$ above the dentate line so as to remove the whole aganglionic bowel segment. $\underline{13}$

We commenced our dissection posteriorly, as no important pelvic structures were encountered in that location. In cases, where the macroscopic transition zone was visible, frozen section biopsies send from the colon above the transition zone. When there was no visible transition zone was found then we send tissue from dilated, proximal colon for frozen section biopsy about $5 \mathrm{~cm}$ above the suspected transition zone, to see the presence of ganglion cell and absent of hypertrophied nerve trunks (<40 microns).

In this series, there were no anastomotic leak and disruption. No one experiences stricture or pelvic abscess. Enterocolitis is one of the most feared complications that can occur in the early postoperative period following surgery for HD. Because of its severity, we are very aggressive regarding its management. While there is no precise definition of enterocolitis, we begin treatment with rectal irrigations and metronidazole when any of our postoperative patients develop abdominal distention, fever, abdominal pain and/ or foulsmelling loose stool. Rectal dilatation is performed by parents if indicated. Irrigation and metronidazole are continued until the episode is resolved and then weaned for a period of 3-4 weeks following the acute episode. We feel this aggressive approach avoids many hospital admissions. Though only three patients of our series required treatment for episodes of enterocolitis. Thus our overall rate of enterocolitis approaches $20 \%$, which is on par with data following a Swenson procedure and the 18$20 \%$ quoted a rate of enterocolitis following the Soave or Duhamel procedure. $\underline{3,4,17,18,20}$

From a historical perspective, beginning as far back as 1950, there are manuscripts describing the dangers of the Swenson dissection and the risks it poses to pelvic structures as well as the long-term risks of urinary and sexual function. However, there seems to be an absence of scientific data confirming these assertions. Sharman et al reviewed 880 postoperative Swenson operations and found no patients who had urinary incontinence or sexual dysfunction. 17 Dr. Swenson described none who have complained of the aforementioned complications and stresses as we have affirmed a meticulous dissection precisely on the rectal wall 17 as key to avoiding complications. We surmise that such complications occur if the rectal dissection is performed too wide.

Urinary continence was noted in all patients in our series who were old enough to be assessed and agerelated milestones for both urinary and fecal continence were not delayed. Bowel function is a primary end point in managing patients with HD. All of the patients that we assessed for bowel function, who were at least three years old, 
Patients with transanal endo-rectal full through (transanal Soave's) operation may develop constriction by muscle cuff, causing functional obstruction leading to stasis, colonic dilatation, fecal soiling, and bowel emptying problems. $\underline{23}$ The Swenson-like full thickness dissection technique that we employ avoids leaving behind Hirschsprung's bowel altogether except for the preserved 1 $\mathrm{cm}$ above the dentate line. We think there is a great value not to leave behind a muscular cuff.23 It appears there is little, if any, data to suggest that a full-thickness rectal dissection is less safe than other techniques and we believe there are key advantages, including excellent continence and a more pure operation without residual cuff or pouch left which can lead to obstruction.

\section{Conclusion}

Modification of Swenson's original transabdominal dissection concept using the recently describe transanal approach is an excellent technique for HD and produces excellent long-term outcome for fecal and urinary function.

\section{References}

1. Swenson O, Rheinlander, Diamond I. Hirschsprung's disease: A new concept of etiology. N Engl J Med. 1949, 241: $552-56$

2. Swenson O, Bill AH. Resection of rectum and rectosigmoid with preservation of sphincter for benign spastic lesions producing megacolon: An experimental study. Surgery 1948; 24: 212.

3. Soave F. A new operation for the treatment of Hirschsprung's disease. Surgery 1964; 56: 1007-14.

4. Duhamel B. A new operation for the treatment of Hirschsprung's disease. Arch Dis Child. 1960; 35: 3839.

5. Rehbein F, Von Zimmermann H. Results with abdominal resection in Hirschsprung disease. Arch Dis Child. 1960; 35: 29-37.

6. Teitelbaum DH. Coran AG. Primary pull-through for Hirschsprung's disease. Semin Neonatol. 2003; 8: 223 41.

7. De la torre-Mondragon L, Ortega-Salgado JA. Transanal endorectal pull-through for Hirschsprung's disease. J Pediatr Surg. 1998; 33: 1283-86.

8. Langer JC, Minkes Rk, Mazziotti MV, et al: Trans anal one-stage Soave procedure for infants with Hirschsprung's disease. J Pediatr Surg. 1999; 34: 148-52.

9. Georgeson KE, Fuenfer MM, Hardin WD. Primary laparoscopic pull-through for Hirschsprung's disease in infants and children. J Pediatr Surg. 1995; 30: 1017-22.

10. Langer JC, Seifert M, Minkes RK. One stage Soave pullthrough for Hirschsprung's disease: A comparison of the trans anal VS open approaches. J Pediatr Surg. 2000: 35: 820-22.

11. Langer JC, Durrant AC, de LA Torre L, et al. One stage trans anal Soave pull-through for Hirschsprung's disease: A multicenter experience with 141 children. Ann Surg. 2003; 238: 569-83.

12. Rintala RJ. Transanal colo-anal pull-through with a short muscular cuff for classic Hirschsprung's disease. Eur J Pediatr Surg. 2003; 13:181-86.

13. Weidner BC, Wald hausen JH. Swenson revisited: A one -stage transanal pull-through procedure for Hirschsprung's disease. J Pediatric Surg. 2003: 38:120811.

14. Mahajan JK. Rathod KK. Bawa M, Narasinham KL. Transanal Swenson's operation for recto-sigmoid Hirschsprung's disease. Afr J Peditr Surg. 2011; 8: 30105.

15. Levitt MA, Pena A. Imperforate anus and cloacal malformation. In: Ashcraft's pediatric surgery. Holcomb III GW, Murphy JP, Ostlie DJ (eds). $5^{\text {th }}$ edi. 2010, pp 468-90.

16. Sweson O. Follow up on 200 patients treated for Hirschsprung disease during ten years period. Ann Surg. 1957; 146; 706-14.

17. Sherman JO, Syndev ME, Weitzman J, et al. A 40 years multinational retrospective study of 880 Swenson procedures. J Paediatr Surg. 1989; 24: 833-38.

18. Ahmed N, Longeb Je. Evaluation of technique in the transanal pull-through for Hirschsprungs disease: Effect on the outcome. J Paediatr Surg. 2007; 42; 36-40.

19. VonLeeuwen K, Geigeb JD, Barnet JL, Coran AC, Teitelbauwon DH. Stooling and manometric findings after primary pull-through in Hirschsprungs disease: Perineal vs abdominal approaches. Paediatr Surg Int. 2002; 37: 132-35.

20. Holsehnide AM, Borner W, Burman O, Caffarena P. Fetal and electromanometric investigation of postoperative continenece in Hirschsprung disease: An international workshop. Z Kinderchir. 1980; 29: 39-48.

21. Falchetti D, Desant A, Villaneei V, Iannuccelli M. Laparoscopic relief of obstructing folded muscular cuff after transanal pull-through for aganglionosis. Surg Endosc. 2004; 18: 717-18.

22. Wester T, Rintala RJ. Early outcome of transanal pullthrough with short muscle cuff during the neonatal period. J Paediatr Surg. 2004; 39: 157-60.

23. Levitt MA, Dickie B, Pena A. Evaluation and treatment of the patient with Hirchsprung's disease who is not doing well after pull-through procedure. Semin Paediatr Surg. 2010; 19: 146-53. 